\title{
RANGE BASED SENSOR NODE LOCALIZATION IN THE PRESENCE OF UNKNOWN CLOCK SKEWS
}

\author{
Mohammad Reza Gholami ${ }^{\dagger}$ Sinan Gezici ${ }^{\sharp}$, and Erik G. Ström ${ }^{\dagger}$. \\ $\dagger$ Chalmers University of Technology, Department of Signals and Systems, Gothenburg, Sweden \\ $\sharp$ Bilkent University, Department of Electrical and Electronics Engineering, Ankara, Turkey
}

\begin{abstract}
We deal with the positioning problem based on two-way time-ofarrival (TW-TOA) measurements in asynchronous wireless sensor networks. The optimal estimator for this problem poses a difficult global optimization problem. To avoid the drawbacks in solving the optimal estimator, we use approximations and derive linear models, which facilitate efficient solutions. In particular, we employ the least squares method and solve a general trust region subproblem to find a coarse estimate. To further refine the estimate, we linearize the measurements and obtain a linear model which can be solved using regularized least squares. Simulation results illustrate that the proposed approaches asymptotically attain the Cramér-Rao lower bound.

Index Terms- Positioning, two-way time-of-arrival (TW-TOA), trust region subproblem, regularised least squares.
\end{abstract}

\section{INTRODUCTION}

Range based positioning using two-way time-of-arrival (TW-TOA) measurements is a popular technique in the literature. Despite its robustness against an unknown clock offset, TW-TOA based positioning suffers from imperfect clock skews and unknown processing time, so-called the turn-around times [1,2].

A huge number of algorithms have been considered in the literature to address the positioning problem based on TW-TOA measurements in fully or partially synchronized networks. For example, the maximum likelihood estimator [3], linear least-squares [4], squaredrange least squares [5], projection onto convex sets [6-8], and convex relaxation techniques $[9,10]$ have been proposed for synchronized networks. Assuming an unknown turn-around time, authors in $[11,12]$ formulated the positioning problem based on TW-TOA as nonconvex programming and introduced suboptimal estimators to solve the problem. A few researchers tackled the positioning problem in asynchronous networks and proposed various solutions. For instance the authors in [1] studied the TW-TOA based positioning problem when imperfect clock skew is present in both target and reference nodes and employed a least squares approach. The previously proposed approaches need modifications to be effectively applied to the positioning problem in which clock skew and turn around times are also unknown.

In this study, we consider the positioning of a single target node based on TW-TOA measurements for an asynchronous network. A target node transmits a signal to a reference node located at a known position and the reference node responds to the received signal after

This work was supported in part by the Swedish Research Council (contract no. 2007-6363) and in part by the European Commission in the framework of the FP7 Network of Excellence in Wireless COMmunications COMmunications \# (contract no. 318306). an unknown turn-around time delay. As it is common in the literature, we assume that the reference node measures the turn-around time by a loop back test and transmits the estimate to the target node $[13,14]$. The target node then computes the round-trip delay based on an estimate of the turn-around time. The optimal estimator for the positioning problem in the presence of unknown clock skews in the target and reference nodes involves nonconvex optimization and therefore difficult to solve. Using approximations and preprocessing on data, we reformulate the problem by a linear model in which the elements of the unknown parameter vector are dependent on each other. We then employ two techniques based on general trust region subproblem and least squares to solve the problem. With an estimate of the clock skew and the location, we use the measurement once more and linearize it using the first order Taylor series expansion and obtain a linear model. Then, we refine the estimate using regularized least squares. Note that besides different approaches in formulating the problem in [1] and the current study, the technique proposed in [1] is similar to the linear least squares estimator proposed in the coarse estimation step, except for a correction term introduced in this study. In fact, the fine step introduced in this work improves the performance of the estimator proposed in [1]. Moreover, the trust region subproblem method is applied for the first time in this study to the TW-TOA based positioning in the presence of clock skews. Simulation results show that the proposed approaches asymptotically attain the Cramér-Rao lower bound.

In summary the main contributions of this study are:

- the MLE for the approximate TW-TOA measurement model to find the location and clock skew of the target node;

- two suboptimal estimators based on squared-range least squares to provide coarse estimates of the location and the clock skew;

- a refining approach to improve the coarse estimate provided by the suboptimal estimators.

\section{SYSTEM MODEL}

Consider a two dimensional network with $N$ reference (anchor) nodes located at known positions $\mathbf{a}_{i}=\left[\begin{array}{ll}a_{i, 1} & a_{i, 2}\end{array}\right]^{T} \in \mathbb{R}^{2}$, $i=1, \ldots, N$. Suppose that one target node is placed at unknown position $\mathbf{x}=\left[\begin{array}{ll}x_{1} & x_{2}\end{array}\right]^{T} \in \mathbb{R}^{2}$. We assume that the target node estimates the distance to a reference node by performing a TW-TOA measurement. That is, the target node sends a signal to a reference node and the reference node responds to the received signal after a turn-around time. We assume that the clocks of sensor nodes follow an affine model $[15,16]$. Therefore, the TW-TOA measurement between the target and reference node $i$ can be expressed as [1]

$$
z_{i}=f\left(\frac{d_{i}}{c}+\frac{T_{i}}{2}\right)+\frac{n_{i}}{2}
$$


where $f$ is the clock skew of the target node, $d_{i}=\left\|\mathbf{x}-\mathbf{a}_{i}\right\|$ is the Euclidean distance between reference node $i$ and the point $\mathbf{x}, c$ is the propagation speed, and $T_{i}$ is the turn around time at reference node $i$ in response to the signal transmitted by the target node, $n_{i}$ is the TOA estimation error, which is commonly modeled as a zeromean Gaussian random variable, i.e., $n_{i} \sim \mathcal{N}\left(0, \sigma_{i}^{2}\right)$. One way to deal with the unknown parameter $T_{i}$ is to jointly estimate it along with the location of the target node [11]. It can also be estimated by reference node $i$ using a loop back test and is sent back to the target node [14]. In this study, we consider the latter approach. Suppose an estimate of $T_{i}$ is expressed as

$$
\hat{T}_{i}=f_{i} T_{i}+\epsilon_{i}
$$

where $f_{i}$ and $\epsilon_{i}$ are the clock skew and the TOA estimation error, respectively, at reference node $i$. We assume that $\epsilon_{i}$ is a zero mean Gaussian random variable, i.e., $\epsilon_{i} \sim \mathcal{N}\left(0, \gamma_{i}^{2}\right)$.

Combining (1) and (2), we arrive at the following model:

$$
z_{i}-f \frac{\hat{T}_{i}}{2}=f \frac{d_{i}}{c}+\frac{T_{i}}{2}\left(f-f f_{i}\right)+\frac{n_{i}}{2}-f \frac{\epsilon_{i}}{2} .
$$

We can further simplify the model in (3) as follows. Since in practical scenarios clock skews $f$ and $f_{i}$ are close to one [16], the second term at the right-hand-side of (3) is negligible for a large network in which the turn around time is small. Then, we can approximate the model in (3) as

$$
z_{i} \simeq f \frac{d_{i}}{c}+f \frac{\hat{T}_{i}}{2}+\frac{n_{i}}{2}-f \frac{\epsilon_{i}}{2} .
$$

Throughout the paper, we work with model in (4). We collect the measurements in vector $\mathbf{z}$ as follows:

$$
\mathbf{z}=\left[z_{1} \ldots z_{N}\right]^{T} .
$$

Based on the model in (4) besides the position of the target node, one also needs to estimate the clock skew $f$.

\section{MAXIMUM LIKELIHOOD ESTIMATOR}

In order to obtain the MLE for joint estimation of the position and clock skew of the target node, the following optimization problem needs to be solved [17]:

$$
\left[\hat{f} \hat{\mathbf{x}}^{T}\right]=\underset{\mathbf{x} \in \mathbb{R}^{2}, f \in \mathbb{R}}{\arg \max } p_{\mathbf{Z}}(\mathbf{z} ; f, \mathbf{x}),
$$

where $p_{\mathbf{Z}}(\mathbf{z} ; f, \mathbf{x})$ is the probability density function (pdf) of vector $\mathbf{z}$ indexed by the vector $\left[f \mathbf{x}^{T}\right]$. Since the TOA errors are assumed to be independent and identically distributed random variables, the pdf of $\mathbf{z}$ can be calculated from (4) and (5) as

$$
\begin{aligned}
& p_{\mathbf{Z}}(\mathbf{z} ; f, \mathbf{x}) \\
& =\prod_{i=1}^{N} \sqrt{\frac{8}{\pi\left(\sigma_{i}^{2}+f^{2} \gamma_{i}^{2}\right)}} \exp \left(-\frac{2\left(z_{i}-f d_{i} / c-f \hat{T}_{i} / 2\right)^{2}}{\left(\sigma_{i}^{2}+f^{2} \gamma_{i}^{2}\right)}\right) .
\end{aligned}
$$

After some manipulations, the MLE formulation can be expressed as

$$
\begin{aligned}
{\left[\begin{array}{ll}
\hat{\mathbf{x}}^{T} & \hat{f}
\end{array}\right]^{T}=\underset{\mathbf{x} \in \mathbb{R}^{2}, f \in \mathbb{R}}{\operatorname{argmin}} } & \sum_{i=1}^{N} \frac{4}{\left(\sigma_{i}^{2}+f^{2} \gamma_{i}^{2}\right)}\left(z_{i}-f \frac{\hat{T}_{i}}{2}-f \frac{d_{i}}{c}\right)^{2} \\
& +\ln \left(\sigma_{i}^{2}+f^{2} \gamma_{i}^{2}\right) .
\end{aligned}
$$

As observed from (8), the MLE problem is highly nonconvex and therefore is difficult to solve. In the next section, we propose two suboptimal estimators followed by a refining step to solve the positioning problem in the presence of an unknown clock skew.

\section{PROPOSED TECHNIQUES}

In this section, we propose a two step estimation approach to find estimates of the target location. In the proposed procedure, we first obtain coarse estimates of the target location. In the next step, we refine the estimates.

\subsection{Coarse estimate}

In this section, we propose two techniques based on squared-range least squares and obtain a coarse estimate. We first divide both sides of (4) by $f$ and express the model as

$$
z_{i} \alpha-\frac{\hat{T}_{i}}{2}=\frac{d_{i}}{c}+\frac{n_{i}}{2} \alpha-\frac{\epsilon_{i}}{2},
$$

where $\alpha=1 / f$. In the following, the model in (9) is employed to derive the proposed suboptimal estimators. We assume that the measurement noise $n_{i} / 2 \alpha-\epsilon_{i} / 2$ is small compared to $d_{i} / c$. Then, taking the square of both sides of (9) and dropping the small terms yield

$$
\left(z_{i} \alpha\right)^{2}+\frac{\hat{T}_{i}^{2}}{4}-z_{i} \hat{T}_{i} \alpha \simeq \frac{1}{c^{2}}\left(\mathbf{x}^{T} \mathbf{x}-2 \mathbf{a}_{i}^{T} \mathbf{x}+\left\|\mathbf{a}_{i}\right\|^{2}\right)+\nu_{i},
$$

where $\nu_{i}=d_{i}\left(\alpha n_{i}-\epsilon_{i}\right) / c$.

\subsubsection{General trust region subproblem (GTR)}

We first apply a weighted least squares criterion to the model in (10) and obtain the following minimization problem:

$$
\begin{aligned}
& \underset{\mathbf{y}}{\operatorname{minimize}}\left\|\mathbf{W}^{-1 / 2}(\mathbf{A y}-\mathbf{b})\right\|^{2} \\
& \text { subject to } \mathbf{y}^{T} \mathbf{D} \mathbf{y}+2 \mathbf{f}^{T} \mathbf{y}=0
\end{aligned}
$$

where matrices $\mathbf{W}, \mathbf{A}$, and $\mathbf{D}$, and vectors $\mathbf{b}, \mathbf{f}$, and $\mathbf{y}$ are defined as

$$
\begin{aligned}
& \mathbf{A} \triangleq\left[\begin{array}{cccc}
\frac{1}{c^{2}} & -\frac{2}{c} \mathbf{a}_{1} & -z_{1}^{2} & z_{1} \hat{T}_{1} \\
\vdots & \vdots & \vdots & \vdots \\
\frac{1}{c^{2}} & -\frac{2}{c} \mathbf{a}_{N} & -z_{N}^{2} & z_{N} \hat{T}_{N}
\end{array}\right], \quad \mathbf{f} \triangleq\left[\begin{array}{c}
-\frac{1}{2} \\
0 \\
0 \\
-\frac{1}{2} \\
0
\end{array}\right], \\
& \mathbf{D} \triangleq\left[\begin{array}{ccccc}
0 & 0 & 0 & 0 & 0 \\
0 & 1 & 0 & 0 & 0 \\
0 & 0 & 1 & 0 & 0 \\
0 & 0 & 0 & 0 & 0 \\
0 & 0 & 0 & 0 & 1
\end{array}\right], \quad \mathbf{b} \triangleq\left[\begin{array}{c}
-\frac{1}{c^{2}}\left\|\mathbf{a}_{1}\right\|^{2}+\frac{\hat{T}_{1}^{2}}{4} \\
\vdots \\
-\frac{1}{c^{2}}\left\|\mathbf{a}_{N}\right\|^{2}+\frac{\hat{T}_{N}^{2}}{4}
\end{array}\right], \\
& \mathbf{W}=\operatorname{diag}\left(d_{1}^{2}\left(\sigma_{1}^{2}+f^{2} \gamma_{1}^{2}\right), \ldots, d_{N}^{2}\left(\sigma_{N}^{2}+f^{2} \gamma_{N}^{2}\right)\right), \\
& \mathbf{y} \triangleq\left[\|\mathbf{x}\|^{2} \mathbf{x}^{T} \alpha^{2} \alpha\right]^{T} .
\end{aligned}
$$

The problem in (11) is called a generalized trust region subproblem (GTR) [18] and can be solved exactly. It has also been known that the GTR has zero duality gap and the optimal solution can be extracted from the dual solution [18-20]. A necessary and sufficient condition for $\mathbf{y}^{*}$ to be optimal in (9) is that there exist a $\mu \in \mathbb{R}$ [19]

$$
\begin{aligned}
& \left(\mathbf{A}^{T} \mathbf{W}^{-1} \mathbf{A}+\mu \mathbf{D}\right) \mathbf{y}^{*}=\left(\mathbf{A}^{T} \mathbf{W}^{-1} \mathbf{b}-\mu \mathbf{f}\right), \\
& \left(\mathbf{y}^{*}\right)^{T} \mathbf{D} \mathbf{y}^{*}+2 \mathbf{f}^{T} \mathbf{y}^{*}=0, \quad\left(\mathbf{A}^{T} \mathbf{W}^{-1} \mathbf{A}+\mu \mathbf{D}\right) \succeq 0 .
\end{aligned}
$$

Under the conditions in (13), the solution to the problem in (11) is given by

$$
\mathbf{y}(\mu)=\left(\mathbf{A}^{T} \mathbf{W}^{-1} \mathbf{A}+\mu \mathbf{D}\right)^{-1}\left(\mathbf{A}^{T} \mathbf{W}^{-1} \mathbf{b}-\mu \mathbf{f}\right) .
$$


In such a situation to find $\mu$, we simply replace (14) into constraint $\mathbf{y}^{T} \mathbf{D y}+2 \mathbf{f}^{T} \mathbf{y}=0$, i.e.,

$$
\phi(\mu)=\mathbf{y}^{T}(\mu) \mathbf{D} \mathbf{y}^{T}(\mu)+2 \mathbf{f}^{T} \mathbf{y}(\mu)=0, \quad \mu \in \mathcal{I}
$$

where the interval $\mathcal{I}$ consists of all $\mu$ such that $\mathbf{A}^{T} \mathbf{W}^{-1} \mathbf{A}+\mu \mathbf{D} \succeq 0$. The interval of $\mathcal{I}$ is given by [5] $\mathcal{I}=\left(-1 / \mu_{1}, \infty\right)$ with $\mu_{1}$ representing the largest eigenvalue of $\left(\mathbf{A}^{T} \mathbf{W}^{-1} \mathbf{A}\right)^{-1 / 2} \mathbf{D}\left(\mathbf{A}^{T} \mathbf{W}^{-1} \mathbf{A}\right)^{-1 / 2}$ [18]. In summary, the solution to (11) is obtained as follows:

- Use a bisection search to find a root of $\phi(\mu)=0$, say $\mu^{*}$. Note that $\phi(\mu)$ is a strictly decreasing function [18].

- Replace $\mu^{*}$ into (14) to obtain $\mathbf{y}^{*}=\mathbf{y}\left(\mu^{*}\right)$.

- Estimate the unknown location as $\hat{\mathbf{x}}=\left[\mathbf{y}^{*}\right]_{2: 3}$, with $[\mathbf{v}]_{i: j}$ denoting the $i$ th to the $j$ th elements of vector $\mathbf{v}$.

Note that since the weighting matrix $\mathbf{W}$ depends on the unknown distance $d_{i}$, we first replace $\mathbf{W}$ with the identity matrix and find an estimate of the location. Then, we form an approximate weighting matrix from (12) with estimates of $d_{i}$ and $f$ from the first iteration. In fact the procedure explained in the bullet list above is executed twice.

\subsubsection{Linear Least squares (LLS)}

In this section we obtain an LLS solution similar to those in [12,21]. We consider the following linear model (obtained from (10)):

$$
\mathbf{b}=\mathbf{A y}+\boldsymbol{\nu}
$$

where $\boldsymbol{\nu}=\left[\begin{array}{lll}\nu_{1} & \ldots & \nu_{N}\end{array}\right]^{T}$ and $\mathbf{A}, \mathbf{y}$, and $\mathbf{b}$ are given in (12). The unconstrained least squares solution to (16) assuming that $\mathbf{A}$ has full column rank is given by [17]

$$
\hat{\mathbf{y}}=\left(\mathbf{A}^{T} \mathbf{W}^{-1} \mathbf{A}\right)^{-1} \mathbf{A}^{T} \mathbf{W}^{-1} \mathbf{b} .
$$

The covariance matrix of $\hat{\mathbf{y}}$ can be computed as

$$
\mathbf{C}_{\hat{\mathbf{y}}}=\left(\mathbf{A}^{T} \mathbf{W}^{-1} \mathbf{A}\right)^{-1} \text {. }
$$

Note that for a large network, matrix $\mathbf{A}$ is ill-conditioned [12]. Then, we can use the approach introduced in $[12,22]$. In order to further improve the estimate consider the following relations:

$$
\begin{aligned}
& {[\mathbf{y}]_{1}=\|x\|^{2}+\xi_{1}, \quad[\mathbf{y}]_{4}=\alpha^{2}+\xi_{4},} \\
& {[\mathbf{y}]_{2}=x_{1}+\xi_{2}, \quad[\mathbf{y}]_{3}=x_{2}+\xi_{3}, \quad[\mathbf{y}]_{5}=\alpha+\xi_{5},}
\end{aligned}
$$

where $\boldsymbol{\xi}=\left[\begin{array}{lll}\xi_{1} & \ldots & \xi_{5}\end{array}\right]^{T}$ is the estimation error vector. Assuming small estimation errors, we take the squares of the last three equations in (19) and obtain the following expressions:

$$
[\mathbf{y}]_{2}^{2} \simeq x_{1}^{2}+2 x_{1} \xi_{2}, \quad[\mathbf{y}]_{3}^{2} \simeq x_{2}^{2}+2 x_{2} \xi_{3}, \quad[\mathbf{y}]_{5} \simeq \alpha^{2}+2 \alpha \xi_{5}
$$

Based on (19) and (20), we obtain a linear model as

$$
\mathbf{h}=\mathbf{B} \boldsymbol{\theta}+\mathbf{P} \boldsymbol{\xi}
$$

where

$$
\begin{gathered}
\mathbf{B}=\left[\begin{array}{lll}
1 & 1 & 1 \\
1 & 0 & 0 \\
0 & 1 & 0 \\
0 & 0 & 1
\end{array}\right], \quad \mathbf{P}=\left[\begin{array}{ccccc}
1 & 0 & 0 & 0 & 1 \\
0 & 2 x_{1} & 0 & 0 & 0 \\
0 & 0 & 2 x_{2} & 0 & 0 \\
0 & 0 & 0 & 2 \alpha & 0
\end{array}\right] \\
\mathbf{h}=\left[[\mathbf{y}]_{1}+[\mathbf{y}]_{4}[\mathbf{y}]_{2}^{2}[\mathbf{y}]_{3}^{2}[\mathbf{y}]_{4}^{2}\right]^{T}, \quad \boldsymbol{\theta}=\left[x_{1}^{2} x_{2}^{2} \alpha^{2}\right]^{T} .
\end{gathered}
$$

The least squares solution to (21) is given by

$$
\hat{\boldsymbol{\theta}}=\left(\mathbf{B}^{T} \mathbf{C}_{\hat{\boldsymbol{\theta}}}^{-1} \mathbf{B}\right)^{-1} \mathbf{B}^{T} \mathbf{C}_{\hat{\boldsymbol{\theta}}}^{-1} \mathbf{h},
$$

where the covariance matrix $\mathbf{C}_{\boldsymbol{\theta}}$ can be computed as

$$
\mathbf{C}_{\hat{\boldsymbol{\theta}}}=\mathbf{P} \mathbf{C}_{\hat{\mathbf{y}}} \mathbf{P}^{T} \text {. }
$$

To compute matrix $\mathbf{P}$ we use the estimate $\hat{\mathbf{x}}=[\hat{\mathbf{y}}]_{2: 3}$ obtained in (17) instead of unknown vector $\mathbf{x}$.

Finally the location estimate can be obtained as

$$
\tilde{x}_{i}=\operatorname{sgn}\left(y_{i+1}\right) \sqrt{\left|\hat{\boldsymbol{\theta}}_{i}\right|}, \quad i=1,2,
$$

where sgn denotes the signum function.

\subsection{Fine estimate}

In this section, we first refine the estimate of the clock skew. Assuming an estimate of the location $\overline{\mathbf{x}}(\overline{\mathbf{x}}=\hat{\mathbf{x}}$ from GTR or $\overline{\mathbf{x}}=\tilde{\mathbf{x}}$ from LLS), an estimate of the clock skew can be obtained from (4) (using the method of moment [17]) as follows:

$$
\hat{f}=\frac{\sum_{i=1}^{N} z_{i}}{\sum_{i=1}^{N} \bar{d}_{i} / c+\hat{T}_{i} / 2},
$$

where $\bar{d}_{i}=\left\|\overline{\mathbf{x}}-\mathbf{a}_{i}\right\|$. Now applying the first order Taylor series expansion about $\overline{\mathbf{x}}$ and assuming an estimate of the clock skew given in (26), we get the following expression:

$$
z_{i} \simeq \hat{f} \frac{\hat{d}}{c}+\hat{f} \frac{\hat{T}_{i}}{2}+\mathbf{g}_{i}^{T} \Delta \mathbf{x}+\frac{n_{i}}{2}-\hat{f} \frac{\epsilon_{i}}{2},
$$

where $\mathbf{g}_{i}=\hat{f}\left(\overline{\mathbf{x}}-\mathbf{a}_{i}\right) /\left(c \bar{d}_{i}\right)$, and $\Delta \mathbf{x}=\mathbf{x}-\overline{\mathbf{x}}$. Thus, we arrive at the following linear model to estimate the error of estimation $\Delta \mathrm{x}$ :

$$
\mathbf{t}=\mathbf{G} \Delta \mathbf{x}+\boldsymbol{\vartheta}
$$

where $\boldsymbol{\vartheta}=\left[n_{1} / 2-\hat{f} \epsilon_{1} / 2 \ldots n_{N} / 2-\hat{f} \epsilon_{N} / 2\right]^{T}, \mathbf{G}=\left[\mathbf{g}_{1}^{T} \ldots \mathbf{g}_{N}^{T}\right]^{T}$, and $\mathbf{t}=\left[z_{1}-\hat{f}\left(\bar{d}_{1} / c+\hat{T}_{1} / 2\right) \ldots z_{N}-\hat{f}\left(\bar{d}_{N} / c+\hat{T}_{N} / 2\right)\right]^{T}$.

The assumption in deriving the model in (28) is that the error of estimation, i.e., $\Delta \mathbf{x}$, is small enough. We take this assumption into account and apply the regularized least squares (Tikhonov regularization technique) to find an estimate of the $\Delta \mathbf{x}$ as [23]

$$
\hat{\Delta} \mathbf{x}=\left(\mathbf{G}^{T} \mathbf{J}^{-1} \mathbf{G}+\lambda \mathbf{I}_{2}\right)^{-1} \mathbf{G}^{T} \mathbf{J}^{-1} \mathbf{t},
$$

where $\mathbf{J}=\operatorname{diag}\left(\sigma_{1}^{2}+\hat{f} \gamma_{1}^{2}, \ldots, \sigma_{N}^{2}+\hat{f} \gamma_{N}^{2}\right)$ and $\lambda$ defines a trade-off between $(\mathbf{G} \Delta \mathbf{x}-\mathbf{t})^{T} \mathbf{J}^{-1}(\mathbf{G} \Delta \mathbf{x}-\mathbf{t})$ and $\|\Delta \mathbf{x}\|^{2}$ terms [22].

Finally, the updated estimate is obtained as

$$
\hat{\overline{\mathbf{x}}}=\overline{\mathbf{x}}+\hat{\Delta} \mathbf{x} .
$$

A note on complexity analysis: The worst-case complexity for the MLE using the Gauss-Newton method considering a good initial point can be computed as $O\left(K_{G N} N^{3}\right)$, where $K_{G N}$ is the number of iterations (usually less than 50). The corresponding LLS needs an order of $O(52 N)$ to implement. For the GTR, we need to use a bisection search to solve (15), which is the most complex part of the algorithm. Suppose the bisection search takes $k_{2}$ steps (usually 20 to 30), then the total cost of the the proposed approach can be approximated as $O\left(36 k_{2}+52 N\right)$. Note that we need to run the LLS and GTR twice. Hence, the corresponding complexities are 
Table 1. Complexity and average running time of different approaches.

\begin{tabular}{|l|l|l|}
\hline Method & Complexity & Time $(\mathrm{ms})$ \\
\hline \hline MLE (for good starting point) & $O\left(k_{1} N^{3}\right)$ & 196 \\
\hline LLS(coarse) & $O(52 N)$ & 0.36 \\
\hline GTR(coarse) & $O\left(36 k_{2}+52 N\right)$ & 5.5 \\
\hline Fine step & $O(14 N)$ & 0.21 \\
\hline
\end{tabular}

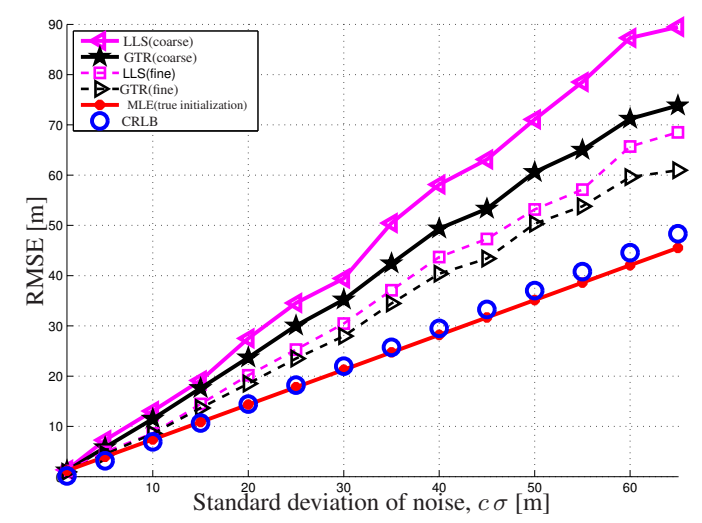

(a)

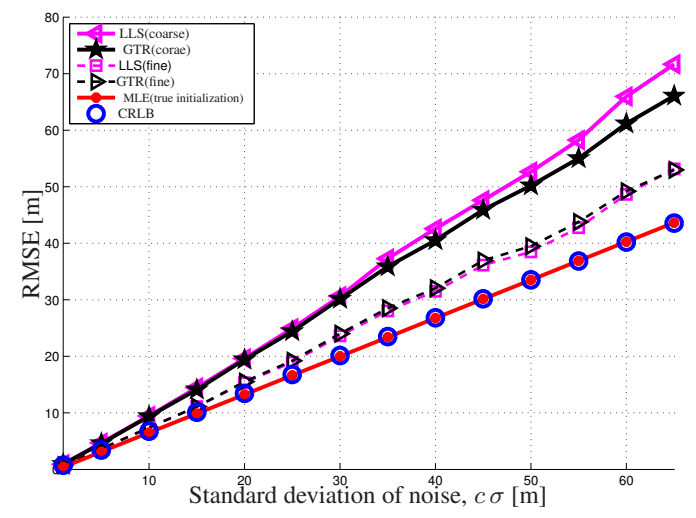

(b)

Fig. 1. The RMSE of difference approaches for (a) 7 reference nodes and (b) 8 reference nodes.

increased by a factor of two. The complexity of the fine step can be computed as $O(14 N)$.

We have also measured the average running time of 500 realizations for a network consisting of seven reference nodes as considered in Section 5. The algorithms have been implemented in Matlab on MacBook Pro (Processor 2.3 GHz Intel Core i7, Memory 8 GB 1600 MHz DDR3). The MLE is implemented by Matlab function lsqnonlin [24] initialized with the true values of the target position and the clock skew. The complexity and average running time are shown in Table 1. From the table, we see a reasonable cost of the proposed approaches for practical implementation.

\section{NUMERICAL RESULTS}

In this section, we evaluate the performance of the proposed approaches through computer simulations. We consider a 800 by 800 square meters area and a number of reference nodes that are located at fixed positions $\mathbf{a}_{1}=\left[\begin{array}{ll}400 & 400\end{array}\right], \mathbf{a}_{2}=\left[\begin{array}{ll}400 & -400\end{array}\right], \mathbf{a}_{3}=$ $\left[\begin{array}{ll}-400400\end{array}\right], \mathbf{a}_{4}=\left[\begin{array}{ll}-400 & -400\end{array}\right], \mathbf{a}_{5}=\left[\begin{array}{ll}400 & 400\end{array}\right], \mathbf{a}_{6}=$ $[0400], \mathbf{a}_{7}=[-4000]$, and $\mathbf{a}_{8}=[0-400]$. In the simulations, we pick the first $n$ reference nodes, i.e., $\mathbf{a}_{1}, \ldots, \mathbf{a}_{n}$. One target node is randomly distributed inside the area. The clock skew and turnaround time are uniformly drawn from [0.99 1.01] and [0 0.001$]$ $\mathrm{ms}$, respectively. We compare the proposed techniques with the MLE in (8) and the Cramér-Rao lower bound (CRLB) computed in Appendix A. In the simulation, we assume that $\sigma_{i}=\gamma_{i}=\sigma$ for $i=1, \ldots, N$. In addition, we set $\lambda=0.02$.

Fig. 1 shows the root-mean-squares-errors (RMSEs) of location estimaties for different approaches versus the scaled standard deviation of noise, i.e., $c \sigma$, for seven and eight reference nodes. It is observed that the both of the proposed approaches attain the CRLB for low standard deviations of noise. It is also seen that the GTR based approach achieves better performance than the LLS.

\section{CONCLUSIONS}

In this paper, we have studied TW-TOA based positioning in the presence of imperfect clock skews and unknown turn-around times. Since the optimal ML estimator is highly nonconvex and difficult to solve, we have used approximations and derived a linear model in which the elements of the unknown vector are dependent on each other. We have applied two techniques based on the general trust region subproblem and the least squares approach. To improve the estimate further, we have linearized measurements around the estimate and applied a regularized least squares approach. Simulation results show that the proposed techniques can attain the CRLB for low standard deviations of noise.

\section{A. CRAMÉR-RAO LOWER BOUND (CRLB)}

Based on (7), the elements of the Fisher information matrix can be computed as [17]

$$
\begin{aligned}
F_{J J}= & -\mathbb{E}\left[\frac{\partial^{2} \ln p_{\mathbf{Z}}(\mathbf{z} ; f, \mathbf{x})}{\partial x_{J}^{2}}\right] \\
& =\sum_{i=1}^{N} \frac{4 f^{2} / c^{2}}{\left(\sigma_{i}^{2}+f^{2} \gamma_{i}^{2}\right)} \frac{\left(x-a_{i, J}\right)^{2}}{d_{i}^{2}}, J=1,2, \\
F_{12}=F_{21}= & -\mathbb{E}\left[\frac{\partial^{2} \ln p_{\mathbf{Z}}(\mathbf{z} ; f, \mathbf{x})}{\partial x_{1} \partial x_{2}}\right] \\
& =\sum_{i=1}^{N} \frac{4 f^{2} / c^{2}}{\left(\sigma_{i}^{2}+f^{2} \gamma_{i}^{2}\right)} \frac{\left(x_{1}-a_{i, 1}\right)\left(x_{2}-a_{i, 2}\right)}{d_{i}^{2}}, \\
F_{33}= & -\mathbb{E}\left[\frac{\partial^{2} \ln p_{\mathbf{Z}}(\mathbf{z} ; f, \mathbf{x})}{\partial f^{2}}\right] \\
& =\sum_{i=1}^{N} \frac{4\left(\hat{T}_{i} / 2+d_{i} / c\right)}{\left(\sigma_{i}^{2}+f^{2} \gamma_{i}^{2}\right)}+4 f^{2} \frac{\gamma_{i}^{2}}{\left(\sigma_{i}^{2}+f^{2} \gamma_{i}^{2}\right)^{2}} \\
F_{3 J}=F_{J 3}= & -\mathbb{E}\left[\frac{\partial^{2} \ln p_{\mathbf{Z}}(\mathbf{z} ; f, \mathbf{x})}{\partial x_{J} \partial f}\right] \\
= & \sum_{i=1}^{N} \frac{f / c\left(\hat{T}_{i} / 2+d_{i} / c\right)}{\sigma_{i}^{2}\left(1+f^{2}\right)} \frac{x-a_{i, 1}}{d_{i}}, J=1,2 .
\end{aligned}
$$

Then, the CRLB, which is a lower bound on the variance of any unbiased estimator, is given by

$$
\begin{aligned}
& \mathbb{E}\left\{\|\hat{\mathbf{x}}-\mathbf{x}\|^{2}\right\} \\
& \geq \frac{F_{33}\left(F_{22}+F_{11}\right)-\left(F_{32}^{2}+F_{13}^{2}\right)}{F_{33}\left(F_{11} F_{22}-F_{12}^{2}\right)+\left(2 F_{31} F_{23} F_{12}-F_{22} F_{13}^{2}-F_{11} F_{23}^{2}\right)} .
\end{aligned}
$$




\section{B. REFERENCES}

[1] Y. Wang, X. Ma, and G. Leus, "Robust time-based localization for asynchronous networks," IEEE Trans. Signal Process., vol. 59, no. 9, pp. 4397-4410, Sep. 2011.

[2] M. R. Gholami, "Positioning algorithms for wireless sensor networks," Licentiate Thesis, Chalmers University of Technology, Mar. 2011. [Online]. Available: http://publications.lib.chalmers.se/records/fulltext/138669.pdf

[3] G. Mao and B. Fidan, Localization Algorithms and Strategies for Wireless Sensor Networks. Information Science reference, Hershey. New York, 2009.

[4] X. Wang, Z. Wang, and B. O'Dea, "A TOA-based location algorithm reducing the errors due to non-line-of-sight (NLOS) propagation," IEEE Trans. Veh. Technol., vol. 52, no. 1, pp. 112-116, Jan. 2003.

[5] A. Beck, P. Stoica, and J. Li, "Exact and approximate solutions of source localization problems," IEEE Trans. Signal Process., vol. 56, no. 5, pp. 1770-1778, May 2008.

[6] A. O. Hero and D. Blatt, "Sensor network source localization via projection onto convex sets (POCS)," in Proc. IEEE International Conference on Acoustics, Speech and Signal Processing, vol. 3, Philadelphia, USA, Mar. 2005, pp. 689-692.

[7] M. R. Gholami, S. Gezici, E. G. Ström, and M. Rydström, "A distributed positioning algorithm for cooperative active and passive sensors," in Proc. IEEE International Symposium on Personal, Indoor and Mobile Radio Communications (PIMRC), Sep. 2010.

[8] M. R. Gholami, H. Wymeersch, E. G. Ström, and M. Rydström, "Wireless network positioning as a convex feasibility problem," EURASIP Journal on Wireless Communications and Networking 2011, 2011:161.

[9] P. Tseng, "Second-order cone programming relaxation of sensor network localization," SIAM J. Optim., vol. 18, no. 1, pp. 156-185, Feb. 2007.

[10] P. Biswas, T.-C. Lian, T.-C. Wang, and Y. Ye, "Semidefinite programming based algorithms for sensor network localization," ACM Trans. Sens. Netw., vol. 2, no. 2, pp. 188-220, 2006.

[11] M. R. Gholami, S. Gezici, E. G. Ström, and M. Rydström, "Positioning algorithms for cooperative networks in the presence of an unknown turn-around time," in Proc. the 12th IEEE International Workshop on Signal Processing Advances in Wireless Communications (SPAWC), San Francisco, Jun. 26-29 2011, pp. 166-170.

[12] M. R. Gholami, S. Gezici, and E. G. Ström, "Improved position estimation using hybrid TW-TOA and TDOA in cooperative networks," IEEE Trans. Signal Process., vol. 60, no. 7, pp. 3770 -3785, Jul. 2012.

[13] S. Gezici and Z. Sahinoglu, "Enhanced position estimation via node cooperation," in Proc. IEEE International Conference on Communications (ICC), Cape Town, South Africa, May 23-27 2010.

[14] Z. Sahinoglu, "Improving range accuracy of IEEE 802.15.4a radios in the presence of clock frequency offsets," IEEE Commun. Lett., vol. 15, no. 2, pp. 244-246, Feb. 2011.
[15] Y.-C. Wu, Q. Chaudhari, and E. Serpedin, "Clock synchronization of wireless sensor networks," IEEE Signal Process. Mag., vol. 28, no. 1, pp. 124-138, Jan. 2011.

[16] E. Serpedin and Q. M. Chaudhari, Synchronization in wireless sensor networks: parameter estimation, performance benchmarks and protocols. New York, NY, USA: Cambridge University Press, 2009.

[17] S. M. Kay, Fundamentals of Statistical Signal Processing: Estimation theory. Englewood Cliffs, NJ: Prentice-Hall, 1993.

[18] J. J. More, "Generalization of the trust region problem," Optimization Methods and Software, vol. 2, pp. 189-209, 1993.

[19] D. Sorensen, "Newtons method with a model trust region modification," SIAM J. Numer. Anal., vol. 19, no. 2, pp. 409-426, 1982.

[20] A. Beck and Y. C. Eldar, "Strong duality in nonconvex quadratic optimization with two quadratic constraints," SIAM J. on Optimi., vol. 17, no. 3, pp. 844-860, Sep. 2006.

[21] M. Sun and K. C. Ho, "Successive and asymptotically efficient localization of sensor nodes in closed-form," IEEE Trans. Signal Process., vol. 57, no. 11, pp. 4522-4537, Nov. 2009.

[22] S. Boyd and L. Vandenberghe, Convex Optimization. Cambridge University Press, 2004.

[23] M. R. Gholami, S. Gezici, E. G. Ström, and M. Rydström, "Hybrid TW-TOA/TDOA positioning algorithms for cooperative wireless networks," in Proc. IEEE International Conference on Communications (ICC), Kyoto, Japan, Jun. 2011.

[24] The Mathworks Inc., 2012. [Online]. Available: http: //www.mathworks.com 